\title{
The management of Moderate Acute Malnutrition (MAM) in children aged 6- 59 months: A systematic review and meta-analysis
}

Gluning $\mathrm{I}^{1,2}$, Kerac $\mathrm{M}^{1,3}$, Bailey $\mathrm{J}^{1,4}$, Bander $\mathrm{A}^{1}$, Opondo $\mathrm{C}^{5,6}$

Affiliations:

1. Department of Population Health, London School of Hygiene and Tropical Medicine, London, UK

2. Brighton and Sussex University Hospitals Trust, Brighton, UK

3. Centre for MARCH (Maternal, Reproductive, Adolescent \& Child Health Centre), London School of Hygiene and Tropical Medicine, London, UK

4. International Rescue Committee, New York, NY, USA

5. Department of Medical Statistics, London School of Hygiene and Tropical Medicine, London, UK

6. National Perinatal Epidemiology Unit, University of Oxford, Oxford, UK

\section{$\underline{\text { Abstract }}$}

Background: Malnutrition is a leading cause of morbidity and mortality in children aged under five years, especially in low- and middle- income countries (LMICs). Although severe acute malnutrition (SAM) is considered the most serious form of malnutrition, moderate acute malnutrition (MAM) affects greater numbers globally and, unlike SAM, guidelines lack a robust evidence-base. This systematic review and meta-analysis assessed the evidence for lipid-based nutrient supplements (LNS), fortified-blended-flours (FBF) and nutrition counselling, in the treatment of MAM.

Methods: Five databases were systematically searched for studies conducted in LMICs that compared the effectiveness of food-based products versus any comparator group in promoting recovery from MAM in children aged 6-59 months. Where appropriate, pooled estimates of effect were estimated using random-effects meta-analyses.

Results: A total of thirteen trials were identified for inclusion. All used active controls rather than 'standard care', which is often minimal in most settings. There was evidence of increased probability of recovery (as assessed by gaining normal weight-for-height and/or MUAC) among children treated with LNS compared to children treated with FBF (RR 1.05, 95\% Cl 1.01-1.09, $\mathrm{p}=0 \cdot 009)$. Treatment with an LNS was also associated with a lower risk of persistent MAM at the end of treatment compared with a FBF (RR 0.82, 95\% Cl 0.71-0.95, $p=0 \cdot 007)$.

Conclusion: Based on a relatively small number of studies mainly from Africa, LNS are superior to FBF in improving anthropometric recovery from MAM. The true benefit of MAM treatment may be underestimated due to all studies using active controls rather than usual care which is minimal. More high-quality evidence is needed to evaluate nutrition education/counselling alone as a MAM intervention. Studies should also assess a wider range of outcomes including body composition,

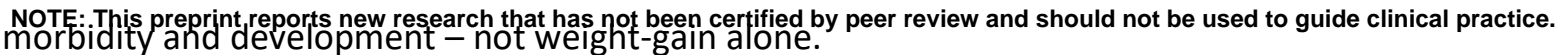


medRxiv preprint doi: https://doi.org/10.1101/2021.01.16.21249861; this version posted January $20,2021$. The copyright holder for this preprint (which was not certified by peer review) is the author/funder, who has granted medRxiv a license to display the preprint in It is made available under a CC-BY 4.0 International license .

\section{$\underline{\text { Abbreviations }}$}

AM Acute Malnutrition

CCC Child Centred Counselling

CCT Controlled-Clinical Trials

$\mathrm{Cl} \quad$ Confidence Interval

ComPAS Combined Protocol for Acute Malnutrition Study

CSB Corn-Soy Blend

FBF $\quad$ Fortified Blended Flours

FFM Fat-Free Mass

FFMI Fat-Free Mass Index

HAZ Height-for-Age z-score

HFIAS Household Food Insecurity Access Scale

HIV Human Immunodeficiency Virus

LMIC Low- and Middle- Income Country

LNS Lipid-based Nutrient Supplement

MAM Moderate Acute Malnutrition

MSBP Malted-Sorghum Based Porridge

MUAC Middle Upper Arm Circumference

NCHS National Centre for Health Statistics 
medRxiv preprint doi: https://doi.org/10.1101/2021.01.16.21249861; this version posted January $20,2021$. The copyright holder for this preprint (which was not certified by peer review) is the author/funder, who has granted medRxiv a license to display the preprint in It is made available under a CC-BY 4.0 International license.

PICO Point, Intervention, Comparator, Outcome

PRISMA Preferred Reporting-Items for Systematic Reviews and Meta-Analyses

RCT Randomised-Controlled Trial

RR Relative Risk

RUF Ready-to-Use Food

RUSF Ready-to-Use Supplementary Food

RUTF Ready-to-Use Therapeutic Food

SAM Severe Acute Malnutrition

SFP Supplementary Feeding Programme

TB Tuberculosis

UN United Nations

UNICEF United Nations Children's Fund

WFP World Food Programme

WHO World Health Organisation

WLZ Weight-for-length z-score

WHZ Weight-for-Height z-score 
medRxiv preprint doi: https://doi.org/10.1101/2021.01.16.21249861; this version posted January $20,2021$. The copyright holder for this preprint (which was not certified by peer review) is the author/funder, who has granted medRxiv a license to display the preprint in It is made available under a CC-BY 4.0 International license.

\section{Introduction}

Malnutrition is a leading cause of morbidity and mortality in children aged under five, especially in low- and middle- income countries (LMIC) ${ }^{1}$. Acute malnutrition (AM) comprises wasting and/or nutritional oedema. Wasting is defined by low weight-for-length/height z-score (WLZ/WHZ) and/or low mid-upper arm circumference (MUAC). AM is often subdivided into severe acute malnutrition (SAM) and moderate acute malnutrition (MAM). In 2019, of the 47 million children under five years of age who were acutely malnourished, $32 \cdot 7$ million suffered from MAM${ }^{1}$.

To date, much focus has been on treating children with SAM since individual case-fatality is higher. However, MAM also matters: it affects greater numbers of children globally; children with MAM have a three-fold increased risk of mortality compared to those without MAM; children with MAM are at-risk of deteriorating to SAM and undergo poorer physical and cognitive development compared to their well-nourished counterparts ${ }^{1,2,3}$.

MAM treatment is context-specific and commonly involves one of two options: 1) improving the adequacy of the home diet through nutrition education/counselling ${ }^{4}$, or 2) supplementary feeding with an energy-dense product. The latter may be necessary in settings with food insecurity or where dietary diversity is poor, and involve targeted supplementary feeding programmes (SFPs) ${ }^{5}$. In recent years, treatment of MAM has predominantly focused on the use of fortified blended flours (FBF) and lipid-based nutrient supplements (LNS) as standard care ${ }^{5,6}$. A key barrier to scale-up is the lack of World Health Organization (WHO) guidelines on MAM. This review aims to inform an upcoming 2021 WHO guideline review where MAM (moderate wasting) is one of four key topics being examined ${ }^{7}$. 
medRxiv preprint doi: https://doi.org/10.1101/2021.01.16.21249861; this version posted January $20,2021$. The copyright holder for this preprint (which was not certified by peer review) is the author/funder, who has granted medRxiv a license to display the preprint in It is made available under a CC-BY 4.0 International license.

\section{Methods}

We followed the PRISMA (Preferred Reporting-Items for Systematic Reviews and Meta-Analyses) reporting framework ${ }^{8}$.

\section{Inclusion Criteria}

Eligibility and inclusion into the review were based on the following PICO (Population, Intervention, Comparator, and Outcome) framework:

- Children aged 6-59 months diagnosed with MAM and living in a LMIC. MAM was defined as having a WHZ $<-2$ and $\geq-3$ based on WHO 2006 Growth Standards ${ }^{9}$, and/or a MUAC $<12 \cdot 5 \mathrm{~cm}$ and $\geq 11.5 \mathrm{~cm}$, without bipedal oedema.

- A supplementary food product used for the treatment of MAM. This includes: FBFs such as corn-soy blend (CSB), LNSs or ready-to-use therapeutic/supplementary food (RUTF/RUSF), or any complementary food supplement to be consumed in addition to the home diet.

- A comparative treatment group containing participants with MAM, who are receiving either: no specific intervention ('usual care' control, since MAM is not routinely identified or treated in all settings); active control, which includes an alternative food/supplement, nutrition education/counselling, any intervention/combination of interventions used to treat MAM.

- The primary outcome of interest was recovery from MAM, defined as having a WHZ >-2, and/or a MUAC $>12.5 \mathrm{~cm}$, without, bipedal oedema, and based on WHO 2006 Growth Standards. Secondary outcomes of interest were; persistent MAM, progression to SAM, death, defaulting, and any adverse-effects to treatment including body composition.

Only published studies with a control/comparator group were included. For generalizability and inter-study comparability, we only included studies from 2006 to present (2020): 2006 marking the development of the WHO 2006 Growth Standards for defining MAM and SAM, taking over the previously used NCHS References.

\section{Search Methods}


medRxiv preprint doi: https://doi.org/10.1101/2021.01.16.21249861; this version posted January $20,2021$. The copyright holder for this preprint (which was not certified by peer review) is the author/funder, who has granted medRxiv a license to display the preprint in It is made available under a CC-BY 4.0 International license.

The following databases were searched by two authors independently, with final search completed on the $29^{\text {th }}$ October 2020: EMBASE (Ovid), MEDLINE (Ovid), CINAHL (EBSCO), Global Health and ClinicalTrials.gov.

Searches were limited to human-only studies, published between the years 2006 and 2020, and written in English language. A detailed search strategy is in Appendix 1.

The reference lists of identified studies were also checked for papers that met inclusion criteria.

\section{Data Collection}

Studies identified in the search were initially screened by title and abstract to determine if they met eligibility criteria. They were then screened from their full text. Reasons for exclusion were documented.

Data was collected using a data collection form (Table 1) generated from the 'checklist of items to consider in data collection or data extraction,' as part of the Cochrane Methods handbook ${ }^{10}$. Additional information was sought on the differences in characteristics of children who recovered from MAM, versus those who did not recover/progressed to SAM/defaulted/died.

\section{Risk of Bias}

Risk of bias was determined using the Cochrane Collaboration's Tool for Assessing Risk of Bias ${ }^{11}$, which considers the following: 1) random sequence generation, 2) allocation concealment, 3) blinding of participants and personnel, 4) blinding of outcome assessment, 5) incomplete outcome data, and 6) selective reporting.

For each criterion, a judgement was made as to whether the trial was at a 'low-risk,' a 'high-risk,' or an 'unclear-risk' of bias (Table 2).

\section{Study Protocol}

We pre-registered the study at www.crd.york.ac.uk/prospero/display record.php?RecordID=68513.

\section{Data Analysis}

For our primary and secondary outcomes we used STATA v $\cdot 14^{12}$ to perform random-effects metaanalyses to estimate pooled risk ratios (RR) for the outcomes. Random-effects meta-analyses were chosen to incorporate the expected random variation in the effect of each intervention across the studies into the pooled estimates. 
medRxiv preprint doi: https://doi.org/10.1101/2021.01.16.21249861; this version posted January 20, 2021. The copyright holder for this preprint (which was not certified by peer review) is the author/funder, who has granted medRxiv a license to display the preprint in It is made available under a CC-BY 4.0 International license.

We also used descriptive synthesis to summarise findings about characteristics of children who recovered from MAM versus those who did not. 
medRxiv preprint doi: https://doi.org/10.1101/2021.01.16.21249861; this version posted January $20,2021$. The copyright holder for this preprint (which was not certified by peer review) is the author/funder, who has granted medRxiv a license to display the preprint in It is made available under a CC-BY 4.0 International license.

\section{$\underline{\text { Results }}$}

Our search (Appendix 1) generated 1,968 references after removing 309 duplicates. Thirteen papers were eligible for inclusion in the systematic review and meta-analysis: Figure 1. Reasons for exclusion of papers included: unpublished results; using NCHS references for defining MAM; using an alternative measurement of MAM (other than WHZ or MUAC); using a different definition for recovery from MAM (i.e. WHZ > -1), and/or investigating MAM prevention rather than MAM treatment (Appendix 2).

\section{Study Characteristics}

Individual study characteristics are presented in Table 1.

Twelve studies were conducted in African countries, the other in Iran ${ }^{13}$. Two were in urban areas; ten in rural settings; one was set in both an urban and rural area.

Sample sizes ranged from 81 to 2,712. Enrollment ages differed (Table 1), though all children were between 6-60 months. As per inclusion criteria, all studies defined MAM with either WHZ based on WHO 2006 Growth Standards and/or MUAC.

All included studies were randomised-controlled trials (RCTs), of which four were cluster-RCTs. The following interventions were compared:

1. Lipid-based nutrient supplements (LNS) versus fortified blended flours (FBF)

2. Comparison of two different formulations of FBF

3. Comparison of two different formulations of LNS

4. A food supplement versus nutrition counselling/usual diet 


\begin{tabular}{|c|c|c|c|c|c|c|c|c|c|c|c|}
\hline \multirow[t]{2}{*}{ Study } & \multicolumn{2}{|c|}{ Methods } & \multicolumn{3}{|c|}{ Participants } & \multirow{2}{*}{$\begin{array}{l}\text { Intervention(s) } \\
\text { Control }\end{array}$} & \multirow[t]{2}{*}{ Outcomes } & \multicolumn{2}{|l|}{ Results } & \multicolumn{2}{|l|}{ Other } \\
\hline & $\begin{array}{l}\text { Study } \\
\text { Design }\end{array}$ & $\begin{array}{l}\text { Study } \\
\text { Duration }\end{array}$ & $\begin{array}{l}n \\
\text { (total) }\end{array}$ & $\begin{array}{l}\text { Country } \\
\text { and } \\
\text { Setting }\end{array}$ & $\begin{array}{l}\text { Ages in } \\
\text { months }\end{array}$ & & & $\begin{array}{l}\text { Analysed } \\
\text { participants }\end{array}$ & $\begin{array}{l}\text { Number (\%) } \\
\text { of } \\
\text { participants } \\
\text { recovered }\end{array}$ & $\begin{array}{l}\text { Authors' } \\
\text { Conclusions }\end{array}$ & Additional Comments \\
\hline $\begin{array}{l}\text { Matilsky } \\
2009\end{array}$ & $\mathrm{RCT}$ & 8 weeks & 1362 & $\begin{array}{l}\text { Rural } \\
\text { Malawi }\end{array}$ & $6-59 m$ & $\begin{array}{l}\text { CSB, } \\
\text { vs. } \\
\text { soy/peanut LNS } \\
\text { vs. } \\
\text { milk/peanut } \\
\text { LNS - all } \\
75 \mathrm{kcal} / \mathrm{kg} / \text { day }\end{array}$ & $\begin{array}{l}\text { Recovery: } \\
\text { WHZ >-2 }\end{array}$ & $\begin{array}{l}\text { CSB (439), } \\
\text { soy/peanut } \\
\text { LNS (438), } \\
\text { milk/ } \\
\text { peanut LNS } \\
(456)\end{array}$ & $\begin{array}{l}\text { CSB, } \\
323(73 \cdot 6 \%) \\
\text { soy/peanut } \\
360(82 \cdot 2 \%) \\
\text { milk/peanut } \\
369(80 \cdot 9 \%)\end{array}$ & $\begin{array}{l}\text { Using LNS over } \\
\text { CSB in SFPs, } \\
\text { milk/protein and } \\
\text { soy/protein are } \\
\text { equally effective }\end{array}$ & $\begin{array}{l}\text { Characteristics of children who } \\
\text { developed oedema: } \\
\text { Younger age, lower WHZ, no } \\
\text { weight gain after } 2 \text { weeks } \\
(p<0 \cdot 001)\end{array}$ \\
\hline $\begin{array}{l}\text { LaGrone } \\
2012\end{array}$ & $\mathrm{RCT}$ & 12 weeks & 2712 & $\begin{array}{l}\text { Rural } \\
\text { Malawi }\end{array}$ & $\begin{array}{l}\text { 6-59 } \\
\text { months }\end{array}$ & $\begin{array}{l}\text { CSB++, } \\
\text { vs. } \\
\text { local soy RUSF, } \\
\text { vs. } \\
\text { soy/whey RUSF } \\
\text { ( } 75 \mathrm{kcal} / \mathrm{kg} / \text { day) }\end{array}$ & $\begin{array}{l}\text { Recovery: } \\
\text { WHZ > -2 }\end{array}$ & $\begin{array}{l}\text { CSB++ } \\
(888), \\
\text { soy RUSF } \\
(906), \\
\text { soy/whey } \\
\text { RUSF (918) }\end{array}$ & $\begin{array}{l}\text { CSB++ } \\
763,(85 \cdot 9 \%) \\
\text { soy RUSF } \\
795(87 \cdot 7 \%) \\
\text { soy/whey } \\
\text { RUSF } \\
807(87 \cdot 9 \%)\end{array}$ & $\begin{array}{l}\text { CSB++ is not } \\
\text { inferior to RUSF }\end{array}$ & $\begin{array}{l}\text { Associated with recovery: child } \\
\text { enrolled in post- harvest (April- } \\
\text { July), able to stand without } \\
\text { assistance, taking antibiotics, } \\
\text { mother has had HIV test, } \\
\text { vomiting in } 2 \text { wks before } \\
\text { enrolment, HAZ, child has had } \\
\text { HIV test, child known as HIV } \\
\text { positive, WHZ, receiving TB } \\
\text { treatment }\end{array}$ \\
\hline $\begin{array}{l}\text { Nikiema } \\
2014\end{array}$ & $\mathrm{RCT}$ & 12 weeks & 1974 & $\begin{array}{l}\text { Rural } \\
\text { Burkina } \\
\text { Faso }\end{array}$ & $\begin{array}{l}6-24 \\
\text { months }\end{array}$ & $\begin{array}{l}\text { CCC, } \\
\text { vs } \\
\text { CSB++ } \\
\text { ( } 273 \mathrm{kcal} / \text { day }) \text {, } \\
\text { vs. } \\
\text { local soy RUSF } \\
\text { ( } 258 \cdot 3 \mathrm{kcal} / \text { day })\end{array}$ & $\begin{array}{l}\text { Recovery: } \\
\text { WHZ > - } 2\end{array}$ & $\begin{array}{l}\text { CCC (605), } \\
\text { CSB++ } \\
\text { (675), RUSF } \\
(694)\end{array}$ & $\begin{array}{l}\text { CCC } \\
350(57 \cdot 9 \%), \\
\text { CSB++ } \\
503(74 \cdot 5 \%), \\
\text { RUSF } \\
515(74 \cdot 2 \%)\end{array}$ & $\begin{array}{l}\text { Supplementary } \\
\text { feeding is better } \\
\text { than nutrition } \\
\text { counselling alone. } \\
\text { However, } \\
\text { nutrition } \\
\text { education is also } \\
\text { important to } \\
\text { treatment }\end{array}$ & $\begin{array}{l}\text { Restricting analysis to non- } \\
\text { defaulters showed no significant } \\
\text { results. Children who developed } \\
\text { SAM were significantly younger, } \\
\text { reported more episodes of } \\
\text { illness in the weeks preceding } \\
\text { their inclusion, had a lower } \\
\text { initial WHZ and had a poorer } \\
\text { attendance. }\end{array}$ \\
\hline
\end{tabular}




\begin{tabular}{|c|c|c|c|c|c|c|c|c|c|c|c|}
\hline $\begin{array}{l}\text { Medoua } \\
2015\end{array}$ & RCT & 12 weeks & 81 & $\begin{array}{l}\text { Urban / } \\
\text { rural } \\
\text { Cameroo } \\
\text { n }\end{array}$ & $\begin{array}{l}6-59 \\
\text { months }\end{array}$ & $\begin{array}{l}\text { CSB+, } \\
\text { vs. } \\
\text { RUSF } \\
(75 \mathrm{kcal} / \mathrm{kg} / \text { day) }\end{array}$ & $\begin{array}{l}\text { Recovery: } \\
\text { WHZ > -2 }\end{array}$ & $\begin{array}{l}\text { CSB+ (41); } \\
\operatorname{RUSF}(40)\end{array}$ & $\begin{array}{l}\text { CSB+ } \\
30(73 \cdot 2 \%), \\
\text { RUSF } \\
34(85 \cdot 0 \%)\end{array}$ & $\begin{array}{l}\text { CSB+ and RUSF } \\
\text { both successfully } \\
\text { treated MAM. } \\
\text { Despite low RUSF } \\
\text { ration, recovery is } \\
\text { comparable to } \\
\text { other studies - } \\
\text { potentially the } \\
\text { effect of nutrition } \\
\text { education. }\end{array}$ & \\
\hline $\begin{array}{l}\text { Amegovu } \\
2015\end{array}$ & $\begin{array}{l}\text { Cluster } \\
\text { RCT }\end{array}$ & 12 weeks & 440 & $\begin{array}{l}\text { Rural } \\
\text { Uganda }\end{array}$ & $\begin{array}{l}\text { 6-59 } \\
\text { months }\end{array}$ & $\begin{array}{l}\text { CSB+ } \\
(1,200 \mathrm{kcal} / \mathrm{day}) \text {, } \\
\text { vs. } \\
\text { sorghum } \\
\text { peanut blend } \\
\text { (SPB) } \\
(1,228 \mathrm{kcal} / \text { day })\end{array}$ & $\begin{array}{l}\text { Recovery: } \\
\text { WHZ > - } 2\end{array}$ & $\begin{array}{l}\text { CSB+ (194), } \\
\text { SPB (198) }\end{array}$ & $\begin{array}{l}\text { CSB+ } \\
169(87 \cdot 1 \%) \\
\text { SPB (181; } \\
91 \cdot 4 \%)\end{array}$ & $\begin{array}{l}\text { Supplementation } \\
\text { with a locally } \\
\text { made LNS (SPB) is } \\
\text { effective in MAM } \\
\text { treatment }\end{array}$ & $\begin{array}{l}\text { Non-response rate was above } \\
10 \% \text { acceptable level (SPHERE) }\end{array}$ \\
\hline $\begin{array}{l}\text { Ackatia- } \\
\text { Armah } \\
2015\end{array}$ & $\begin{array}{l}\text { Cluster } \\
\text { RCT }\end{array}$ & 12 weeks & 1284 & Rural Mali & $\begin{array}{l}6-35 \\
\text { months }\end{array}$ & $\begin{array}{l}\text { RUSF } \\
\text { (500kcal/day), } \\
\text { Vs. } \\
\text { CSB ++ } \\
\text { (501kcal/day), } \\
\text { Vs. } \\
\text { Micronutrient- } \\
\text { fortified cereal- } \\
\text { legume blend, } \\
500 k c a l / d a y), \\
\text { Vs. } \\
\text { LMF (less- } \\
\text { refined cereal- } \\
\text { legume milled } \\
\text { flour mis, } \\
500 k c a l / d a y)+ \\
\text { vit A fortified oil } \\
+ \text { micronutrient } \\
\text { sachets } \\
\text { (Mixme) }\end{array}$ & $\begin{array}{l}\text { Recovery: } \\
\text { WHZ > -2 } \\
\text { or MUAC } \geq \\
125 \mathrm{~mm}\end{array}$ & $\begin{array}{l}\text { RUSF (335), } \\
\text { CSB++ } \\
(342), \\
\text { MI (306), } \\
\text { LMF (281) }\end{array}$ & $\begin{array}{l}\text { RUSF } \\
(73 \cdot 1 \%), \\
\text { CSB++ } \\
(61 \cdot 2 \%), \\
\text { MI }(61 \cdot 1 \%), \\
\text { LMF }(57 \cdot 9 \%)\end{array}$ & $\begin{array}{l}\text { Children in the } \\
\text { RUSF group } \\
\text { gained more } \\
\text { weight, MUAC } \\
\text { and WHZ } \\
\text { compared to MI } \\
\text { and LMF groups. } \\
\text { MI and LMF did } \\
\text { not differ from } \\
\text { each other. } \\
\text { CSB++ was } \\
\text { intermediate } \\
\text { between RUSF } \\
\text { and locally } \\
\text { prepared foods. }\end{array}$ & \\
\hline
\end{tabular}




\begin{tabular}{|c|c|c|c|c|c|c|c|c|c|c|c|}
\hline $\begin{array}{l}\text { Stobaugh } \\
2016\end{array}$ & $\mathrm{RCT}$ & 12 weeks & 2259 & $\begin{array}{l}\text { Rural } \\
\text { Malawi }\end{array}$ & $\begin{array}{l}6-59 \\
\text { months }\end{array}$ & $\begin{array}{l}\text { whey RUSF, } \\
\text { vs. } \\
\text { soy RUSF } \\
\text { (all } 75 \\
\mathrm{kcal} / \mathrm{kg} / \text { day }\end{array}$ & $\begin{array}{l}\text { Recovery: } \\
\text { MUAC } \geq \\
125 \mathrm{~mm}\end{array}$ & $\begin{array}{l}\text { Soy RUSF } \\
(1086), \\
\text { whey RUSF } \\
\text { (1144) }\end{array}$ & $\begin{array}{l}\text { Soy RUSF } \\
960(88 \cdot 4 \%) \\
\text { Whey RUSF } \\
874(76 \cdot 4 \%)\end{array}$ & $\begin{array}{l}\text { Milk protein is } \\
\text { important in } \\
\text { supplementary } \\
\text { products for } \\
\text { MAM }\end{array}$ & \\
\hline $\begin{array}{l}\text { Fabianse } \\
\text { n } 2017\end{array}$ & $\mathrm{RCT}$ & 12 weeks & 1609 & $\begin{array}{l}\text { Rural } \\
\text { Burkina } \\
\text { Faso }\end{array}$ & $\begin{array}{l}6-23 \\
\text { months }\end{array}$ & $\begin{array}{l}\text { CSB-type } \\
\text { products, } \\
\text { Vs. } \\
\text { LNS-type } \\
\text { products } \\
\text { (500kcal/day) } \\
\text { ( } 2 \times 2 \times 3 \text { factorial } \\
\text { trial comparing } \\
\text { variants of the } \\
\text { recipe) }\end{array}$ & $\begin{array}{l}\text { Change in } \\
\text { FFMI (fat- } \\
\text { free mass } \\
\text { index): } \\
\text { WHZ>-2, } \\
\text { MUAC } \geq \\
125 \mathrm{~mm} \\
\text { (as a } \\
\text { secondary } \\
\text { outcome) }\end{array}$ & & & $\begin{array}{l}\text { Supplementation } \\
\text { promotes mainly } \\
\text { fat-free mass } \\
\text { accretion. } \\
\text { LNS promotes } \\
\text { more fat-free } \\
\text { mass accretion } \\
\text { and recovery } \\
\text { compared to CSB. }\end{array}$ & \\
\hline $\begin{array}{l}\text { Kajjura } \\
2019\end{array}$ & $\begin{array}{l}\text { Cluster } \\
\text { RCT }\end{array}$ & 12 weeks & 220 & $\begin{array}{l}\text { Rural } \\
\text { Uganda }\end{array}$ & $\begin{array}{l}6-18 \\
\text { months }\end{array}$ & $\begin{array}{l}\text { MSBP (nutrient- } \\
\text { dense } \\
\text { hydrolysed } \\
\text { malted } \\
\text { sorghum-based } \\
\text { porridge - } \\
675 \mathrm{kcal} / \text { day), } \\
\text { Vs. } \\
\text { CSB+ (standard } \\
\text { care in Uganda } \\
\text { - } 600 \mathrm{kcal} / \text { day) }\end{array}$ & $\begin{array}{l}\text { Recovery: } \\
\text { WHZ > -2 }\end{array}$ & $\begin{array}{l}\text { MSBP } \\
(104) \\
\text { CSB+ (100) }\end{array}$ & $\begin{array}{l}\text { MSBP } \\
94(90 \cdot 4 \%) \\
\text { CSB+ } \\
81(81 \cdot 0 \%)\end{array}$ & $\begin{array}{l}\text { MSBP can be used } \\
\text { as a } \\
\text { supplementary } \\
\text { porridge in the } \\
\text { management of } \\
\text { MAM in Uganda. }\end{array}$ & $\begin{array}{l}\text { Also investigated effect on } \\
\text { anaemia: found no effect on } \\
\text { mean } \mathrm{Hb} \text { levels between } \\
\text { groups. }\end{array}$ \\
\hline $\begin{array}{l}\text { KohIman } \\
\text { n } 2019\end{array}$ & $\mathrm{RCT}$ & 12 weeks & 869 & $\begin{array}{l}\text { Rural } \\
\text { Ghana }\end{array}$ & $\begin{array}{l}6-59 \\
\text { months }\end{array}$ & $\begin{array}{l}\text { A-RUTF (locally } \\
\text { produced } \\
\text { alternative } \\
\text { RUTF - } \\
560 \mathrm{kcal} / 100 \mathrm{~g} \text { ), } \\
\text { Vs } \\
\text { S-RUTF } \\
\text { (standard RUTF) }\end{array}$ & $\begin{array}{l}\text { Recovery: } \\
\text { WHZ >-2, } \\
\text { MUAC } \geq \\
125 \mathrm{~mm}\end{array}$ & $\begin{array}{l}\text { A-RUTF } \\
(443), \\
\text { S-RUTF } \\
(426)\end{array}$ & $\begin{array}{l}\text { A-RUTF } \\
386(87 \cdot 1 \%), \\
\text { S-RUTF } \\
398(93 \cdot 4 \%)\end{array}$ & $\begin{array}{l}\text { A-RUTF is inferior } \\
\text { to S-RUTF. } \\
\text { Potentially linked } \\
\text { to higher } \\
\text { defaulting rate. } \\
\text { Further testing is } \\
\text { warranted. }\end{array}$ & $\begin{array}{l}\text { Trial also compared the effects } \\
\text { of each RUTF on SAM patients. } \\
\text { Noted similar effect of poorer } \\
\text { recovery rates using A-RUTF } \\
\text { compared to S-RUTF }\end{array}$ \\
\hline
\end{tabular}




\begin{tabular}{|c|c|c|c|c|c|c|c|c|c|c|c|}
\hline $\begin{array}{l}\text { Azimi } \\
2020\end{array}$ & RCT & 8 weeks & 100 & $\begin{array}{l}\text { Urban } \\
\text { Iran }\end{array}$ & $\begin{array}{l}24-59 \\
\text { months }\end{array}$ & $\begin{array}{l}\text { RUSF } \\
\text { ( } 75 \mathrm{kcal} / \mathrm{kg} / \mathrm{day}) \\
\text { and nutrition } \\
\text { education, } \\
\text { Vs. } \\
\text { Usual diet (UD) } \\
\text { and nutrition } \\
\text { education }\end{array}$ & $\begin{array}{l}\text { Recovery: } \\
\text { WHZ > -1 }\end{array}$ & $\begin{array}{l}\text { With MAM: } \\
\text { RUSF (23), } \\
\text { UD (14) }\end{array}$ & $\begin{array}{l}\text { RUSF } \\
22(95 \cdot 7 \%), \\
\text { UD } \\
9(64 \cdot 3 \%)\end{array}$ & $\begin{array}{l}\text { RUSF significantly } \\
\text { improved growth } \\
\text { indicators and } \\
\text { clinical symptoms } \\
\text { of diarrhoea and } \\
\text { fever in children } \\
\text { with mild- } \\
\text { moderate acute } \\
\text { malnutrition. }\end{array}$ & \\
\hline $\begin{array}{l}\text { Roediger } \\
2020\end{array}$ & $\mathrm{RCT}$ & 12 weeks & 1737 & $\begin{array}{l}\text { Rural } \\
\text { Malawi }\end{array}$ & $\begin{array}{l}6-59 \\
\text { months }\end{array}$ & $\begin{array}{l}\text { High protein } \\
\text { (HiPro)-RUSF } \\
\text { (75kcal/kg/day) } \\
\text { Vs. } \\
\text { Standard } \\
\text { Control-RUSF } \\
\text { (75kcal/kg/day) }\end{array}$ & $\begin{array}{l}\text { Recovery: } \\
W H Z>-2, \\
M U A C \geq \\
125 \mathrm{~mm}\end{array}$ & $\begin{array}{l}\text { HiPro-RUSF } \\
(860), \\
\text { C-RUSF } \\
(877)\end{array}$ & $\begin{array}{l}\text { HiPro-RUSF } \\
759(88 \%), \\
\text { C-RUSF } \\
766(87 \%)\end{array}$ & $\begin{array}{l}\text { Protein quality } \\
\text { scores does not } \\
\text { correlate with } \\
\text { MAM clinical } \\
\text { recovery. } \\
\text { High rates of } \\
\text { recovery with } \\
\text { both RUSF } \\
\text { products. } \\
\text { Recommend } \\
\text { against using } \\
\text { specific protein } \\
\text { quality guideline } \\
\text { to standardise } \\
\text { RUSF }\end{array}$ & \\
\hline $\begin{array}{l}\text { Bailey } \\
2020\end{array}$ & $\begin{array}{l}\text { Cluster } \\
\text { RCT }\end{array}$ & 12 weeks & 1,903 & $\begin{array}{l}\text { Urban } \\
\text { Kenya } \\
\text { and Rural } \\
\text { South } \\
\text { Sudan }\end{array}$ & $\begin{array}{l}6-59 \\
\text { months }\end{array}$ & $\begin{array}{l}\text { RUTF combined } \\
\text { treatment } \\
\text { (500kcal/day), } \\
\text { Vs. } \\
\text { RUSF standard } \\
\text { care } \\
\text { (500kcal/day) }\end{array}$ & $\begin{array}{l}\text { Recovery: } \\
\text { MUAC } \geq \\
125 \mathrm{~mm} \\
\\
\text { Morbidity, } \\
\text { changes in } \\
\text { body } \\
\text { compositio } \\
\text { n (Mean } \\
\text { fat-free }\end{array}$ & $\begin{array}{l}\text { RUTF (995) } \\
\text { RUSF (908) } \\
\text { RUTF (268), } \\
\text { RUSF (375) }\end{array}$ & $\begin{array}{l}\text { RUTF } 860 \\
(86 \cdot 4 \%) \\
\text { RUSF } 773 \\
(85 \cdot 1 \%) \\
\\
\text { RUTF mean } \\
\text { fat-free } \\
\text { mass } \\
(6 \cdot 25 \mathrm{~kg}) \text {, } \\
\text { RUSF mean } \\
\text { fat-free }\end{array}$ & $\begin{array}{l}\text { Combined } \\
\text { treatment for } \\
\text { SAM and MAM is } \\
\text { non-inferior to } \\
\text { standard care } \\
\text { MAM children } \\
\text { had similar body } \\
\text { compositions } \\
\text { when treated } \\
\text { with RUTF versus } \\
\text { RUSF, with both } \\
\text { groups }\end{array}$ & $\begin{array}{l}\text { Morbidity outcomes in MAM } \\
\text { children treated with RUTF } \\
\text { versus RUSF were not } \\
\text { significantly different, this } \\
\text { included: episodes of diarrhoea, } \\
\text { vomiting, fever, cough and } \\
\text { hospitalisation up to four } \\
\text { months post treatment. }\end{array}$ \\
\hline
\end{tabular}




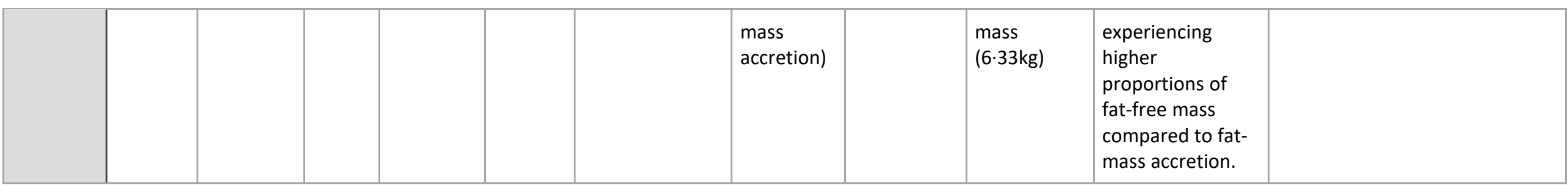

Table 1: Data collection table of included studies

Study characteristics for the thirteen studies included in this systematic review and meta-analysis in order of publication year.

Important abbreviations: CCC, child-centred counselling; CSB, corn-soy blend; HAZ, height-for-age z-score; LNS, lipid-based nutrient supplement; MAM, moderate acute malnutrition; RCT, randomized controlled trial; RUSF, ready to use supplementary food; RUTF, ready to use therapeutic food; SAM, severe acute malnutrition; SFP,

supplementary feeding programme; WHZ, weight-for-height z-score 


\begin{tabular}{|c|c|c|c|c|c|c|c|c|}
\hline Study & $\begin{array}{l}\text { Random } \\
\text { Sequence } \\
\text { Generation }\end{array}$ & $\begin{array}{l}\text { Allocation } \\
\text { Concealment }\end{array}$ & $\begin{array}{l}\text { Blinding of } \\
\text { Participants and } \\
\text { Personnel }\end{array}$ & $\begin{array}{l}\text { Blinding of } \\
\text { Outcome } \\
\text { Assessment }\end{array}$ & $\begin{array}{l}\text { Incomplete } \\
\text { Outcome Data }\end{array}$ & $\begin{array}{l}\text { Selective } \\
\text { Reporting }\end{array}$ & $\begin{array}{l}\text { Other Sources } \\
\text { of Bias }\end{array}$ & $\begin{array}{l}\text { Overall } \\
\text { Assessment }\end{array}$ \\
\hline Matilsky 2009 & Unclear Risk & Low Risk & Unclear Risk & Low Risk & Low Risk & Unclear Risk & Low Risk & Good \\
\hline LaGrone 2012 & Low Risk & Low Risk & Unclear Risk & Low Risk & Low Risk & Unclear Risk & Low Risk & Good \\
\hline Nikiema 2014 & Unclear Risk & Unclear Risk & Unclear Risk & Unclear Risk & High Risk & Unclear Risk & Low Risk & Poor \\
\hline Stobaugh 2016 & Unclear Risk & Low Risk & Low Risk & Low Risk & Low Risk & Unclear Risk & Low Risk & Good \\
\hline Medoua 2015 & Low Risk & Low Risk & Unclear Risk & Low Risk & Low Risk & Unclear Risk & Low Risk & Good \\
\hline Amegovu 2015 & High Risk & Low Risk & Unclear Risk & High Risk & Low Risk & Unclear Risk & Low Risk & Poor \\
\hline Ackatia-A 2015 & Unclear Risk & Unclear Risk & High Risk & Unclear Risk & Low Risk & Unclear Risk & Low Risk & Poor \\
\hline Fabiansen 2017 & Low Risk & Unclear Risk & Unclear Risk & Low Risk & Low Risk & Unclear Risk & Low Risk & Good \\
\hline Kajjura 2019 & Low Risk & Low Risk & Unclear Risk & Low Risk & Low Risk & Unclear Risk & Low Risk & Good \\
\hline Kohlmann 2019 & Low Risk & Low Risk & Low Risk & Low Risk & Unclear Risk & Unclear Risk & Low Risk & Good \\
\hline Azimi 2020 & Low Risk & Unclear Risk & High Risk & Unclear Risk & Low Risk & Low Risk & Low Risk & Good \\
\hline Roediger 2020 & Low Risk & Low Risk & Low Risk & Low Risk & Low Risk & Unclear Risk & Low Risk & Good \\
\hline Bailey 2020 & Low Risk & Low Risk & High Risk & Low Risk & Low Risk & Low Risk & Low Risk & Good \\
\hline
\end{tabular}

Table 1: Risk of bias assessment for the thirteen included studies in order of publication year 
medRxiv preprint doi: https://doi.org/10.1101/2021.01.16.21249861; this version posted January $20,2021$. The copyright holder for this preprint (which was not certified by peer review) is the author/funder, who has granted medRxiv a license to display the preprint in It is made available under a CC-BY 4.0 International license.

All products exceeded WHO energy density recommendations (>0.8kcal/g) ${ }^{14}($ Appendix 3$)$.

The primary outcome of all except two studies was the proportion of children with MAM who recovered. The remaining, investigated increments in the fat-free mass index (FFMI) or fat-mass accretion (kg) of participants ${ }^{15}$. One study did not define recovery as obtaining a $W H Z>-2$, but instead defined recovery as $W H Z>-1^{13}$.

Nevertheless, data was available on the proportions of children who reached a WHZ $>-2$, which was used in this analysis. The four cluster-RCTs reported outcomes adjusted for the clustered design.

In all studies, once a participant was classified as recovered from MAM, they were discharged from the treatment programme. Two studies treated participants for a maximum of 8 weeks ${ }^{16}$; the remaining trials treated for a maximum of 12 weeks.

\section{Risk of Bias}

Table 2 summarises the risk of bias assessment. Overall reporting was good, but with numerous areas unclear in several studies. Randomisation methods in Amegovu $2015^{17}$ were rated as having 'high-risk 'of bias as allocation of the intervention and control was performed on only two clusters. Furthermore, this study specifically reported that the individual assessing outcomes was not blinded to the intervention.

Nikiema $2014^{18}$ experienced a loss to follow-up rate of $44 \cdot 6 \%$ in the nutrition counselling arm, and therefore was considered to have a 'high-risk 'of attrition bias.

Blinding of participants and study personnel was not done in both Ackatia-Armah $2015^{19}$, Azimi 2020 and Bailey $2020^{20}$, due to clear differences between the two interventions. 
medRxiv preprint doi: https://doi.org/10.1101/2021.01.16.21249861; this version posted January $20,2021$. The copyright holder for this preprint (which was not certified by peer review) is the author/funder, who has granted medRxiv a license to display the preprint in perpetuity.

It is made available under a CC-BY 4.0 International license .

\section{Study Results}

\section{Lipid-Based Nutrient Supplement (LNS) vs Fortified Blended Four (FBF)}

Seven trials compared an LNS and a FBF. One study was removed from the final meta-analysis, as it used a type of $\mathrm{FBF}$, corn-soy blend (CSB), which has since been replaced by improved versions $(\mathrm{CSB}+/++)^{16}$. The reason was to reduce heterogeneity. Random-effects meta-analysis of the remaining trials $(n=7,667)$ showed that children treated with an LNS were $4 \%$ more likely to recover with CSB+/++ (RR 1.05, 95\% Cl 1.01-1.09, p=0.009) (Figure 2). Overall, $42 \cdot 1 \%$ of the variation in relative-risks were attributable to heterogeneity $(p=0 \cdot 125)$.

A random-effects meta-analysis of four studies $(n=5,710)$ comparing an LNS with CSB $+/++$, showed that children treated with an LNS have an $18 \%$ reduction in the risk of persistent MAM (RR 0.82, 95\% $\mathrm{Cl} 0 \cdot 71-0.95, p=0 \cdot 009)$. There was no evidence of heterogeneity across studies $(p=0 \cdot 825)$.

Progression to SAM whilst receiving either an LNS or a FBF was reported in five trials $(n=7,043)$. Random-effects meta-analysis showed no significant difference in the risk of developing SAM amongst children treated with an LNS compared to FBF (RR=0.87, 95\%Cl 0.74-1.00, $p=0 \cdot 066)$. There was no evidence of heterogeneity $(p=0 \cdot 879)$.

A random-effects meta-analysis of three trials reporting deaths during treatment for MAM with either an LNS or a $\operatorname{FBF}(n=5,414)$ showed no differences (RR 0.88, 95\% $\mathrm{Cl} 0 \cdot 47-1 \cdot 64, \mathrm{p}=0.687)$. There was also no evidence of heterogeneity across studies $(p=0 \cdot 565)$.

Data from the three trials reporting defaulting from MAM treatment with either an LNS or FBF $(n=5,414)$ showed no evidence of a difference in default rates (RR $1 \cdot 35,95 \% \mathrm{Cl} 0.96-1 \cdot 90, p=0 \cdot 082$ ). There was no evidence of heterogeneity across trials $(p=0 \cdot 307)$.

One study reported no evidence of a difference in the risk of diarrhoea $(p=0.980)$ or vomiting $(p=0 \cdot 220)$ during treatment with an LNS versus a $\mathrm{FBF}^{17}$.

One study investigated fat-free mass accretion after treatment ${ }^{15}$. This showed that children treated with LNS had, on average, $0.083 \mathrm{~kg} / \mathrm{m}^{2}$ higher FFMI than those treated with CSB $(95 \% \mathrm{Cl} 0.003-0 \cdot 163)$. Furthermore, there was no evidence of effect modification for the differences in FFMI by season, admission criteria, baseline FFMI, stunting, inflammation, and breastfeeding.

\section{Different Formulations of LNS}

Seven trials compared two different formulations of $\operatorname{LNS}^{15,16,20,21,22,23,24}$. Detailed compositions of these LNSs is in Appendix 3.

A random-effects meta-analysis of the three trials comparing whey/milk LNS vs soy/no animal product LNS $(n=4,948)$ showed no differences in: recovery (RR 1.01, 95\%Cl 0.98-1.05, $p=0 \cdot 418$, heterogeneity $p=0 \cdot 186)$; risk of 
medRxiv preprint doi: https://doi.org/10.1101/2021.01.16.21249861; this version posted January $20,2021$. The copyright holder for this preprint (which was not certified by peer review) is the author/funder, who has granted medRxiv a license to display the preprint in perpetuity.

It is made available under a CC-BY 4.0 International license.

remaining moderately malnourished $(R R 1 \cdot 02,95 \% \mathrm{Cl} 0 \cdot 77-1 \cdot 36, p=0 \cdot 884$, heterogeneity $p=0 \cdot 515)$; risk of progression to SAM (RR 0.95, 95\% Cl 0.80-1.13, $p=0.559$, heterogeneity $p=0.552)$; risk of death ( $R R 0.8095 \% \mathrm{Cl}$ $0 \cdot 38-1 \cdot 66, p=0 \cdot 542$, heterogeneity $p=0 \cdot 676)$.

Treatment of MAM with a milk-/whey- based LNS was associated with a 39\% lower risk of defaulting comparing to treatment with a soy-LNS ( $n=4,948, R R 0.61,95 \% \mathrm{Cl} 0.40-0.93, p=0.022$ ) (Figure 3). There was no evidence of heterogeneity across trials.

A combined treatment of SAM and MAM was investigated in one study: children with MAM were given one sachet of ready-to-use therapeutic food (RUTF) in the combined protocol, and one sachet of ready-to-use supplementary food (RUSF) in the standard protocol. Treatment of MAM with the combined protocol was noninferior to treatment with standard care $(n=1,903, \mathrm{RR} 0.00,95 \% \mathrm{Cl}-0.07 ; 0.07, p=0.97)^{20}$. At four months posttreatment, there was no significant differences in episodes of diarrhoea (adjusted difference: 0.05, 95\% $\mathrm{Cl}-0.9$; 1.0, $\mathrm{p}=0.91$ ), vomiting (adjusted difference: $0 \cdot 21,95 \% \mathrm{Cl}-1 \cdot 4 ; 1 \cdot 8, \mathrm{p}=0 \cdot 80$ ), fever (adjusted difference: $0.34,95 \% \mathrm{Cl}$ $-0.9 ; 1.6, p=0.59$ ), cough (adjusted difference: $-0.41,95 \% \mathrm{Cl}-1.7 ; 0.9, p=0.53$ ), and hospitalisation (adjusted difference: $0 \cdot 52,95 \% \mathrm{Cl}-1 \cdot 8 ; 2 \cdot 9, \mathrm{p}=0 \cdot 66)^{25}$.

Changes in body composition when treating MAM with two different LNS products was investigated in two studies $^{15}$. One observed that products containing $20 \%$ milk protein were better at promoting fat-free mass accretion than products which did not contain milk protein (FFMI difference: $0.097 \mathrm{~kg} / \mathrm{m}^{2}, 95 \% \mathrm{Cl}-0.002 ; 0 \cdot 196$ ). There was no evidence of an effect on FFMI when treating MAM with products that contained $50 \%$ milk protein compared to no milk protein (FFMI difference: $0.049 \mathrm{~kg} / \mathrm{m}^{2}, 95 \% \mathrm{Cl}-0.047 ; 0.146$ ). At four month follow-up post MAM treatment, no significant differences were observed in fat-free mass accretion when treating with RUTF versus RUSF (adjusted difference: $-0 \cdot 10 \mathrm{~kg}, 95 \% \mathrm{Cl}-0 \cdot 31 ; 0 \cdot 11, \mathrm{p}=0 \cdot 37$ ) ${ }^{25}$.

When considering protein quality, one trial noted no association between LNS protein quality and recovery from $\operatorname{MAM}(p=0.61)^{24}$.

In one study, treating MAM using a locally produced LNS (A-RUTF), which substituted half the amount of peanut in standard LNS (S-RUTF) with local soybean and sorghum flours, was associated with significantly lower proportions of children recovering : LNS (A-RUTF: 386 (87.1), S-RUTF: 398 (93.4), p=0.003) $)^{23}$. One reason for this was thought to be higher defaulting amongst children supplemented with locally produced LNS (A-RUTF: 56 (12.6), S-RUTF: $27(6 \cdot 3), p=0 \cdot 002)$.

\section{Different Formulations of FBF}

One study investigated treating of MAM with two different formulations of a FBF; CSB+ and a nutrient-dense malted sorghum-based porridge (MSBP) in Uganda ${ }^{26}$. MSBP substituted a portion of maize in the commercially 
medRxiv preprint doi: https://doi.org/10.1101/2021.01.16.21249861; this version posted January $20,2021$. The copyright holder for this preprint (which was not certified by peer review) is the author/funder, who has granted medRxiv a license to display the preprint in It is made available under a CC-BY 4.0 International license.

produced CSB+ with malted sorghum. Recovery from MAM did not differ significantly in children treated with MSBP versus CSB+ ( $n=94(90 \%)$ vs $n=81(81 \%), p=0 \cdot 055)$.

\section{Food Supplement vs Nutrition Education / Counselling}

Two studies compared treatment of MAM with a food supplement versus a non-food supplement ${ }^{13,18}$. One compared child-centered counselling (CCC) with a blended food (CSB++) and LNS (RUSF) in Burkina Faso; the other compared an LNS (RUSF) with usual diet and nutrition education in Iran.

Children treated with a food product had an $18 \%$ increased probability of recovery versus children treated without a supplement (Figure 4) (RR 1.16, 95\% Cl 1.02-1·31, p=0.045). There was evidence of heterogeneity across studies $(p=0 \cdot 045)$.

In Azimi 2020 no children progressed to SAM or died and only one child defaulted.

In Nikiema 2014 children treated with a food product had a $22 \%$ reduced risk of progressing to SAM compared to those treated with $C C C(\operatorname{RR~} 0 \cdot 78,95 \% \mathrm{Cl} 0 \cdot 62-0.99, p=0.037)$. There was also strong evidence that children treated with a food product had a $71 \%$ reduced risk of defaulting compared to children treated with CCC (RR $0 \cdot 29,95 \% \mathrm{Cl}$ $0 \cdot 17-0 \cdot 48, p<0 \cdot 001)$. Restriction of the analysis to non-defaulters noted the following proportions of children who recovered in each intervention arm: $71 \cdot 0 \%$ recovered with CCC; $77 \cdot 6 \%$ recovered using CSB++ arm; $79 \cdot 6 \%$ recovered using $\mathrm{RUSF}^{18}$.

There was no evidence for a difference in the proportions of children who remained moderately malnourished (RR 0.97, 95\% Cl 0.78-1.21, p=0.786), or died (RR 0.44, 95\% Cl 0.17-1.17, $p=0 \cdot 101$ ) when treated for MAM with either a food product or CCC.

A significantly lower prevalence of both diarrhoea ( $n=6(12 \%)$ vs $n=14(28 \cdot 6 \%), p=0 \cdot 01)$, and fever (8 (16\%) vs 18 (36.7\%), $p=0.05$ ) was observed in children treated with an LNS compared to usual diet ${ }^{13}$. 
medRxiv preprint doi: https://doi.org/10.1101/2021.01.16.21249861; this version posted January 20, 2021. The copyright holder for this preprint (which was not certified by peer review) is the author/funder, who has granted medRxiv a license to display the preprint in perpetuity.

It is made available under a CC-BY 4.0 International license .

\section{Discussion}

This was a systematic review and meta-analysis investigating the effectiveness of treatments for moderate acute malnutrition (MAM) in children aged 6-59 months in LMICs. Ten of the thirteen included trials compared the effectiveness of different supplementary food products in promoting MAM recovery, reflecting the recent focus on the use of products in MAM management ${ }^{6}$. Children treated with an LNS, had a $5 \%$ increased probability of recovery compared to those treated with CSB++. Furthermore, treatment with an LNS was associated with a lower risk of persistent MAM, compared with a FBF. Treatment with a food product was associated with a higher probability of recovery, a lower risk of progressing to SAM, and a lower risk of defaulting, compared to children who did not receive supplementation.

In the past, others have also reviewed MAM management ${ }^{27,28,29,30,31}$. Results for our primary outcome - recovery - are consistent with others' findings that treating MAM with an LNS was associated with an increased relative risk of recovery, compared to treatment with FBFs: Lazzerini 2013 (RR 1.04, 95\% Cl 0.99-1.09), Lenters 2013 (RR 1·11, 95\%Cl 1·04-1·18), Gera 2017 (RR 1·08, 95\%Cl 1·02-1·14), and Das 2020 (RR 1·07, 95\%Cl 1·02-1·13). All reported relatively few studies, often with significant heterogeneity between studies even after attempts at stratification and subset analysis. The major step forward we took was to only include papers defining MAM using the WHO 2006 Growth Standards (rather than the old NCHS References. This affects the profile of children enrolled and explains greater homogeneity in our results overal132. Since WHO 2006 Growth Standards are now common worldwide, our approach also better represents the current population of interest and our findings enable more generalisable conclusions to be drawn. Another key benefit of our review is to present latest research in this area - hence critical to the upcoming $2021 \mathrm{WHO}$ guidelines process ${ }^{7}$.

The following may contribute to the lower recovery rates seen amongst MAM children treated with FBFs compared with LNSs: 1) FBFs resemble staple foods and thus may be more openly shared amongst other household members ${ }^{33,34,35}$. 2) Children must consume roughly eight-times the mass of FBF compared to LNS, thus potentially discouraging breastfeeding / eating other foods provided at home ${ }^{33,35}$. 3) FBFs are lower in fat content and energy density compared to LNSs (Appendix 2). This is supported by evidence showing that LNS supplementation does not replace other foods in the diet ${ }^{36,37}$, is highly acceptable in an African context ${ }^{38,39}$, and is shared amongst household members substantially less than $\mathrm{FBFs}^{40,41}$.

This review has highlighted some key gaps in the current body of evidence, which can be grouped into population, intervention/comparator and outcome related.

Out of thirteen included studies, twelve were conducted in African populations, making it difficult to generalise conclusions to South Asian contexts. This is problematic given that over half of all wasted children globally live in South Asia ${ }^{1}$, and likely have different growth trajectories and energy needs compared to children 
medRxiv preprint doi: https://doi.org/10.1101/2021.01.16.21249861; this version posted January $20,2021$. The copyright holder for this preprint (which was not certified by peer review) is the author/funder, who has granted medRxiv a license to display the preprint in perpetuity.

It is made available under a CC-BY 4.0 International license .

from Africa ${ }^{20}$. Though promising early evidence highlights the effectiveness and acceptability of LNSs in South Asia ${ }^{42}$, more research is needed to understand similarities and differences compared with other populations.

Another striking feature of studies in our review is that all used active controls. This risks underestimating the true benefit of MAM treatment since the field reality is that children with MAM are neither identified not get any treatment in many settings worldwide. Future studies should consider more 'usual care' controls (i.e. minimal/no specific care) to better understand true impact of MAM care. This would be ethically acceptable in some settings in light of a recent recommendation that children with MAM presenting to primary care should not be routinely treated ${ }^{43}$. Cost-effectiveness data would be another important part of such work.

In eleven included trials, anthropometry and in particular recovery of 'normal' weight was the main outcome measure. Whilst anthropometry is widely used as the key measure of nutritional status, it is malnutrition-associated risk of mortality and morbidity that really matters rather than body size alone ${ }^{44}$. Wasting, low WHZ, which defines MAM is strongly associated with high risk of mortality ${ }^{45,46}$. However, there is increasing evidence that weight recovery does not necessarily mean return to low clinical risk of those who were never malnourished: children who have had MAM remain at high risk of relapse post treatment ${ }^{47}$. Children with MAM, who recovered as part of the LaGrone 2012 study, were followed-up for a further 12-month period in order to assess the long-term effects of treatment with CSB++ and RUSF ${ }^{48}$. During this period, only $63 \%$ of children remained well-nourished, highlighting the vulnerability of children post MAM recovery according to anthropometric measures alone. Measuring the proportions of children who progress to SAM, require admission or die during treatment, are more meaningful measurements of short-term clinical effectiveness of interventions, as these highlights unwanted negative outcomes along the spectrum of anthropometric status. However, these outcomes are relatively rare, can be more complex to measure and certainly in the case of mortality require far sample sizes for robust analysis - so are not commonly included in research.

Other more clinically meaningful outcome measures include body composition and it is encouraging to see studies in our review starting to assess this ${ }^{49}$. Body composition is especially valuable since it also gives information about potential longer term future risk, including of $N C D^{50}$. There is concern that routine supplementation of MAM children with energy-dense products will encourage unhealthy weight-gain that risks predisposition to $\mathrm{NCDs}^{51}$. Balancing this evidence from one of the studies in our review suggests the opposite; that the majority of weight gain during supplementation with an energy-dense product is fat-free mass ${ }^{15}$, and that there are no significant differences in changes in body composition depending on the energy density of products $^{25}$. Both these findings persist up to four months post treatment of MAM ${ }^{25}$.

Another important consideration in the treatment of MAM, is its relation to SAM treatment. Although both conditions lie on a continuum of acute malnutrition, both are managed in separate programmes, using different food products and under two organisations; UNICEF for SAM and WFP for MAM. A cluster-RCT based in Kenya 
medRxiv preprint doi: https://doi.org/10.1101/2021.01.16.21249861; this version posted January 20, 2021. The copyright holder for this preprint (which was not certified by peer review) is the author/funder, who has granted medRxiv a license to display the preprint in It is made available under a CC-BY 4.0 International license .

and South Sudan investigated a combined protocol for treating acute malnutrition compared to standard care ${ }^{20}$. They noted no significant difference between recovery rates (risk difference $0 \cdot 03,95 \% \mathrm{Cl}-0 \cdot 05-0 \cdot 10, \mathrm{p}=0 \cdot 52$ ), and cost-effectiveness of the combined protocol (US\$123 less per child recovered) compared to standard care. These results are echoed in Maust $2015^{52}$. Overall, combined management may be simpler, more cost-effective and reach more children.

We acknowledge some limitations of our review and meta-analysis: 1) Relevant papers could have been missed by restricting the search to papers published after 2006 and limiting to English language. 2) In the risk of bias assessment, we did not search for original paper protocols when determining selective reporting, and therefore, it is unclear as to how much reporting bias contributed to concealment of undesirable results. This was not considered an issue for the primary outcome, recovery, as all papers provided relevant data. 3) We only found a small number of eligible papers, and thus generalisability may be limited, particularly as most papers were conducted in African LMICs. Nevertheless, the small quantity of papers identified highlights the need for more robust research in MAM management, specifically those that address points raised in this review. 
medRxiv preprint doi: https://doi.org/10.1101/2021.01.16.21249861; this version posted January $20,2021$. The copyright holder for this preprint (which was not certified by peer review) is the author/funder, who has granted medRxiv a license to display the preprint in It is made available under a CC-BY 4.0 International license.

\section{Conclusion}

How best to treat children with MAM is a key question for global child health. We found most current evidence focuses on the use of food supplements and involves studies with active controls rather than no-treatment control as is often the case in everyday practice. This risks underestimating the true benefits of treatment. In studies we identified, supplementation with LNS improves anthropometric recovery and prevents progression to SAM compared to supplementation with FBF. The role of nutrition counselling/education alone, or in combination with a supplementary food-product, warrants further research, particularly in areas with good food security. Outcomes in current research focus on weight-recovery: future trials should include more clinically-meaningful outcomes, such as progression to SAM, admission, death and/or changes in body composition.

\begin{tabular}{|l|l|}
\hline $\begin{array}{l}\text { Author } \\
\text { Contributions }\end{array}$ & $\begin{array}{l}\text { Gluning, I-designed the search strategy, conducted the search and meta-analysis, and } \\
\text { wrote the initial manuscript. } \\
\text { Kerac M - conceived the project, supervised and provided feedback on manuscript } \\
\text { drafts. } \\
\text { Bander A - conducted a second search and review of references } \\
\text { Bailey J - provided feedback on manuscript drafts. } \\
\text { Opondo C - conceived the project, supervised, assisted in meta-analysis and provided } \\
\text { feedback on drafts of manuscript } \\
\text { All authors: read and approve of final version of the manuscript }\end{array}$ \\
\hline $\begin{array}{l}\text { Declaration of } \\
\text { Interest }\end{array}$ & \begin{tabular}{l} 
The authors have declared that no competing interests exist. \\
\hline Funding
\end{tabular} \\
\hline
\end{tabular}


medRxiv preprint doi: https://doi.org/10.1101/2021.01.16.21249861; this version posted January $20,2021$. The copyright holder for this preprint (which was not certified by peer review) is the author/funder, who has granted medRxiv a license to display the preprint in perpetuity.

It is made available under a CC-BY 4.0 International license .

\section{$\underline{\text { References }}$}

1 UNICEF/WHO/The World Bank Group joint child malnutrition estimates: levels and trends in child malnutrition: key findings of the 2020 edition. https://www.who.int/publications/i/item/jme2020-edition (accessed Oct 30, 2020).

2 Black RE, Victora CG, Walker SP, et al. Maternal and child undernutrition and overweight in lowincome and middle-income countries. Lancet. 2013; 382: 427-51.

3 James $\mathrm{P}$, Sadler $\mathrm{K}$, Wondafrash $\mathrm{M}$, et al. Children with moderate acute malnutrition with no access to supplementary feeding programmes experience high rates of deterioration and no improvement: Results from a prospective cohort study in rural Ethiopia. PLoS One 2016; 11. DOI:10.1371/journal.pone.0153530.

4 A. A, Ashworth A, Ferguson E. Dietary counseling in the management of moderate malnourishment in children. Food Nutr. Bull. 2009; 30: S405-33.

Annan RA, Webb P, Brown R. Management of Moderate Acute Malnutrition (MAM): Current Knowledge and Practice Collaborating to improve the management of acute malnutrition worldwide. 2014. https://www.ennonline.net/attachments/2289/MAM-management-CMAMForum-Technical-Brief-Sept-2014.pdf (accessed May 25, 2017). Navarro-Colorado C, Mason F, Shoham J. Measuring the Effectiveness of Supplementary Feeding Programmes in Emergencies. Overseas Development Institute, 2008 http://files.ennonline.net/attachments/883/measuring-the-effectiveness-of-sfp-odinetworkpaper063.pdf (accessed July 8, 2017). Call for authors - Scoping review for WHO guideline on the prevention and treatment of wasting in infants and children. https://www.who.int/news-room/articles-detail/call-for-authors-scopingreview-guideline-prevention-treatment-of-wasting-infant-children (accessed Dec 10, 2020). Liberati A, Altman DG, Tetzlaff J, et al. The PRISMA Statement for Reporting Systematic Reviews and Meta-Analyses of Studies That Evaluate Health Care Interventions: Explanation and Elaboration. PLoS Med 2009; 6: e1000100. WHO Child Growth Standards: Methods and Development. http://www.who.int/childgrowth/standards/Technical_report.pdf?ua=1 (accessed July 9, 2017). Cochrane Handbook for Systematic Reviews of Interventions | Cochrane Training. http://training.cochrane.org/handbook (accessed Aug 11, 2017). 
medRxiv preprint doi: https://doi.org/10.1101/2021.01.16.21249861; this version posted January 20,2021 . The copyright holder for this preprint (which was not certified by peer review) is the author/funder, who has granted medRxiv a license to display the preprint in perpetuity.

It is made available under a CC-BY 4.0 International license .

11 Higgins JPT, Altman DG, Gøtzsche PC, et al. The Cochrane Collaboration's tool for assessing risk of bias in randomised trials. BMJ 2011; 343. http://www.bmj.com/content/343/bmj.d5928 (accessed Aug 11, 2017).

12 StataCorp. STATA 14. 2015.

13 Azimi F, Esmaillzadeh A, Alipoor E, Moslemi M, Yaseri M, Hosseinzadeh-Attar MJ. Effect of a newly developed ready-to-use supplementary food on growth indicators in children with mild to moderate malnutrition. Public Heal 2020; 185: 290-7.

14 Supplementary foods for the management of moderate acute malnutrition in infants and children 6-59 months of age. http://apps.who.int/iris/bitstream/10665/75836/1/9789241504423_eng.pdf (accessed July 10, 2017).

15 Fabiansen C, Yamé Ogo CW, luel-Brockdorf A-S, et al. Effectiveness of food supplements in increasing fat-free tissue accretion in children with moderate acute malnutrition: A randomised 2 $\times 2 \times 3$ factorial trial in Burkina Faso. DOI:10.1371/journal.pmed.1002387.

16 Matilsky DK, Maleta K, Castleman T, Manary MJ. Supplementary Feeding with Fortified Spreads Results in Higher Recovery Rates Than with a Corn/Soy Blend in Moderately Wasted Children. J Nutr 2009; 139: 773-8.

17 Amegovu AK, Ochola S, Ogwok P, Yiga P, Musalima J, Juliana M. Efficacy of sorghum peanut blend and corn soy blend plus in the treatment of moderate acute malnutrition in children aged 6-59 months in Karamoja, Uganda: a cluster randomized trial. Nutr. Diet. Suppl. 2014; 6: 75-84.

Nikiema L, Huybregts L, Kolsteren $\mathrm{P}$, et al. Treating moderate acute malnutrition in first-line health services: an effectiveness cluster-randomized trial in Burkina Faso. Am J Clin Nutr 2014; 100: 241-9.

Ackatia-Armah RS, McDonald CM, Doumbia S, Erhardt JG, Hamer DH, Brown KH. Malian children with moderate acute malnutrition who are treated with lipid-based dietary supplements have greater weight gains and recovery rates than those treated with locally produced cereal-legume products: A community-based, cluster-randomized trial. Am J Clin Nutr 2015; 101: 632-45.

20 Bailey J, Opondo C, Lelijveld N, et al. A simplified, combined protocol versus standard treatment for acute malnutrition in children 6-59 months (ComPAS trial): A cluster-randomized controlled non-inferiority trial in Kenya and South Sudan. PLoS Med 2020; 17: 1-22.

21 LaGrone LN, Trehan I, Meuli GJ, et al. A novel fortified blended flour, corn-soy blend \&quot;plusplus,\&quot; is not inferior to lipid-based ready-to-use supplementary foods for the treatment of moderate acute malnutrition in Malawian children. Am J Clin Nutr 2012; 95: 212-9. 
medRxiv preprint doi: https://doi.org/10.1101/2021.01.16.21249861; this version posted January $20,2021$. The copyright holder for this preprint (which was not certified by peer review) is the author/funder, who has granted medRxiv a license to display the preprint in perpetuity.

It is made available under a CC-BY 4.0 International license .

22 Stobaugh HC, Ryan KN, Kennedy JA, et al. Including whey protein and whey permeate in ready-touse supplementary food improves recovery rates in children with moderate acute malnutrition: a randomized, double-blind clinical trial. Am J Clin Nutr 2016; 103: 926-33.

23 Kohlmann K, Callaghan-Gillespie M, Gauglitz JM, et al. Alternative Ready-To-Use Therapeutic Food Yields Less Recovery Than the Standard for Treating Acute Malnutrition in Children From Ghana. Glob Heal Sci Pract 2019; 7: 203-14.

24 Roediger R, Callaghan-Gillespie M, Blackman JK, KohImann K, Maleta K, Manary M. Comparison of two ready-to-use supplementary foods (RUSF) of differing protein quality for the treatment of moderate acute malnutrition (MAM) in Malawi: a randomise, double-blinded, clinical effectiveness trial. Gastroenterology 2020; 158: S-994.

25 Lelijveld N, Musyoki EN, Adongo SW, et al. Relapse and post-discharge body composition of children treated for acute malnutrition using a simplified, combined protocol: a nested cohort from the ComPAS RCT. Peer Rev.

26 Kajjura RB, Veldman FJ, Kassier SM. Effect of a novel supplementary porridge on the nutritional status of infants and young children diagnosed with moderate acute malnutrition in Uganda: a cluster randomised control trial. J Hum Nutr Diet 2019; 32: 295-302.

27 Lazzerini M, Rubert L, Pani P, et al. Specially formulated foods for treating children with moderate acute malnutrition in low- and middle-income countries. Cochrane database Syst Rev 2013; 6: CD009584.

Lenters L, Wazny K, Webb P, Ahmed T, Bhutta ZA. Treatment of severe and moderate acute malnutrition in low- and middle-income settings: a systematic review, meta-analysis and Delphi process. BMC Public Health. 2013; 13: S23.

29 Gera T, Pena-Rosas JP, Boy-Mena E, Sachdev HS. Lipid based nutrient supplements (LNS) for treatment of children (6 months to 59 months) with moderate acute malnutrition (MAM): A systematic review. PLoS One 2017; 12: e0182096.

30 Lelijveld N, Beedle A, Farhikhtah A, Elrayah EE, Bourdaire J, Aburto N. Systematic review of the treatment of moderate acute malnutrition using food products. Matern Child Nutr 2020; 16. DOI:10.1111/mcn.12898.

31 Das JK, Salam RA, Saeed M, Kazmi FA, Bhutta ZA. Effectiveness of Interventions for Managing Acute Malnutrition in Children under Five Years of Age in Low-Income and Middle-Income Countries: A Systematic Review and Meta-Analysis. Nutrients 2020; 12: 116. 
medRxiv preprint doi: https://doi.org/10.1101/2021.01.16.21249861; this version posted January $20,2021$. The copyright holder for this preprint (which was not certified by peer review) is the author/funder, who has granted medRxiv a license to display the preprint in perpetuity.

It is made available under a CC-BY 4.0 International license .

standards in nutrition programmes: Secondary data analysis. Br Med J 2007; 334: 733-5.

33 Medoua GN, Ntsama PM, Christine A, et al. Recovery rate of children with moderate acute malnutrition treated with ready-to-use supplementary food (RUSF) or improved corn-soya blend (CSB+): a randomized controlled trial. DOI:10.1017/S1368980015001238.

34 Shoham J, Duffield A. Proceedings of the World Health Organization/UNICEF/World Food Programme/United Nations high commissioner for refugees consultation on the management of moderate malnutrition in children under 5 years of age. Food Nutr. Bull. 2009; 30: S464-74. Langlois B, Griswold S, Cliffer I, et al. Behavioral Factors Related to Use of Specialized Nutritious Foods in a MAM Treatment Program in Sierra Leone. Curr Dev Nutr 2020; 4: 857-857. Flax VL, Siega-Riz AM, Reinhart GA, Bentley ME. Provision of lipid-based nutrient supplements to Honduran children increases their dietary macro- and micronutrient intake without displacing other foods. Matern Child Nutr 2015; 11 Suppl 4: 203-13.

37 Arimond $\mathrm{M}$, Zeilani M, Jungjohann S, et al. Considerations in developing lipid-based nutrient supplements for prevention of undernutrition: experience from the International Lipid-Based Nutrient Supplements (iLiNS) Project. Matern Child Nutr 2015; 11 Suppl 4: 31-61.

Phuka J, Ashorn U, Ashorn P, et al. Acceptability of three novel lipid-based nutrient supplements among Malawian infants and their caregivers. Matern Child Nutr 2011; 7: 368-77.

Cohuet S, Marquer C, Shepherd S, et al. Intra-household use and acceptability of Ready-to-UseSupplementary-Foods distributed in Niger between July and December 2010. Appetite 2012; 59: 698-705.

40 Karakochuk CD, van den Briel T, Stephens D, Zlotkin S. Food Sharing Practices in Households Receiving Supplemental Foods for the Treatment of Moderate Acute Malnutrition in Ethiopian Children. J. Hunger Environ. Nutr. 2015; 10: 343-55.

41 Wang RJ, Trehan I, LaGrone LN, Weisz AJ, Thakwalakwa CM, Maleta KM. Investigation of food acceptability and feeding practices for lipid nutrient supplements and blended flours used to treat moderate malnutrition. J. Nutr. Educ. Behav. 2013; 45: 258-63.

42 Christian P, Shaikh S, Shamim AA, et al. Effect of fortified complementary food supplementation on child growth in rural Bangladesh: A cluster-randomized trial. Int. J. Epidemiol. 2015; 44: 186276.

43 WHO. WHO | Supplementary foods for the management of moderate acute malnutrition in children. WHO. 2015. http://www.who.int/elena/titles/food_children_mam/en/ (accessed Feb 16, 2017). 
medRxiv preprint doi: https://doi.org/10.1101/2021.01.16.21249861; this version posted January $20,2021$. The copyright holder for this preprint (which was not certified by peer review) is the author/funder, who has granted medRxiv a license to display the preprint in It is made available under a CC-BY 4.0 International license.

44 Kerac M, McGrath M, Connell N, et al. 'Severe malnutrition': Thinking deeplyS, communicating simply. BMJ Glob. Heal. 2020; 5: 3023.

45 Chen LC, Chowdhury A, Huffman SL. Anthropometric assessment of energy-protein malnutrition and subsequent risk of mortality among preschool aged children. Am J Clin Nutr 1980; 33: 183645.

46 Pelletier DL. The relationship between child anthropometry and mortality in developing countries: Implications for policy, programs and future research. J Nutr 1994; 124.

DOI:10.1093/jn/124.suppl_10.2047S.

47 Stobaugh HC, Bollinger LB, Adams SE, et al. Effect of a package of health and nutrition services on sustained recovery in children after moderate acute malnutrition and factors related to sustaining recovery: a cluster-randomized trial. Am J Clin Nutr 2017; published online June 14.

DOI:10.3945/ajcn.116.149799.

$48 \mathrm{CY}$ C, Trehan I, RJ W, et al. Children successfully treated for moderate acute malnutrition remain at risk for malnutrition and death in the subsequent year after recovery. J Nutr 2013; 143: 21520.

49 Owino VO, Murphy-Alford AJ, Kerac M, et al. Measuring growth and medium- and longer-term outcomes in malnourished children. Matern. Child Nutr. 2019; 15. DOI:10.1111/mcn.12790.

50 Wells JCK. Using Body Composition Assessment to Evaluate the Double Burden of Malnutrition. Ann. Nutr. Metab. 2019; 75: 103-8.

51 Grobler L, Visser M, Young T, Schoonees A, Siegfried N. Benefits and harms of supplementary food in moderately under-nourished children. Cape Town, South Africa, 2015 https://www.who.int/maternal_child_adolescent/documents/grobler-supplementary-foodsundernutrition-children-2015.pdf (accessed Dec 10, 2020).

52 Maust A, Koroma AS, Abla C, et al. Severe and Moderate Acute Malnutrition Can Be Successfully Managed with an Integrated Protocol in Sierra Leone. J Nutr 2015; 145: 2604-9. 
medRxiv preprint doi: https://doi.org/10.1101/2021.01.16.21249861; this version posted January 20, 2021. The copyright holder for this preprint (which was not certified by peer review) is the author/funder, who has granted medRxiv a license to display the preprint in It is made available under a CC-BY 4.0 International license .

\section{Figures}

Figure 1: Search Flow Diagram

2,276 references generated through searching databases

- MEDLINE: 863

- EMBASE: 1113

- CINAHL: 276

- Cinicaltrials.gov: 24

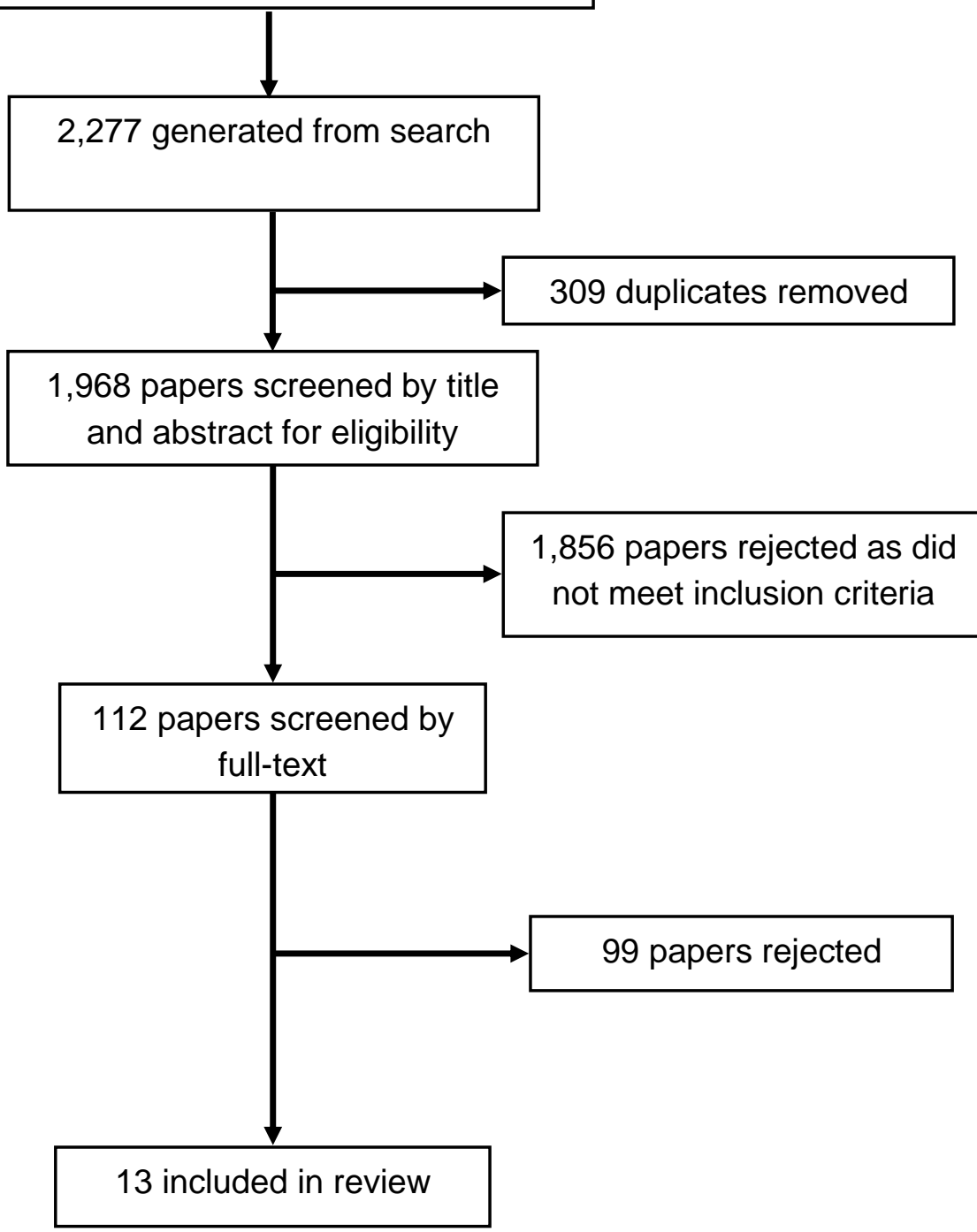


medRxiv preprint doi: https://doi.org/10.1101/2021.01.16.21249861; this version posted January 20, 2021. The copyright holder for this preprint (which was not certified by peer review) is the author/funder, who has granted medRxiv a license to display the preprint in It is made available under a CC-BY 4.0 International license .

Figure 2: A random-effects meta-analysis of studies comparing the proportions of children who recovered from MAM when treated with LNS vs CSB+/++

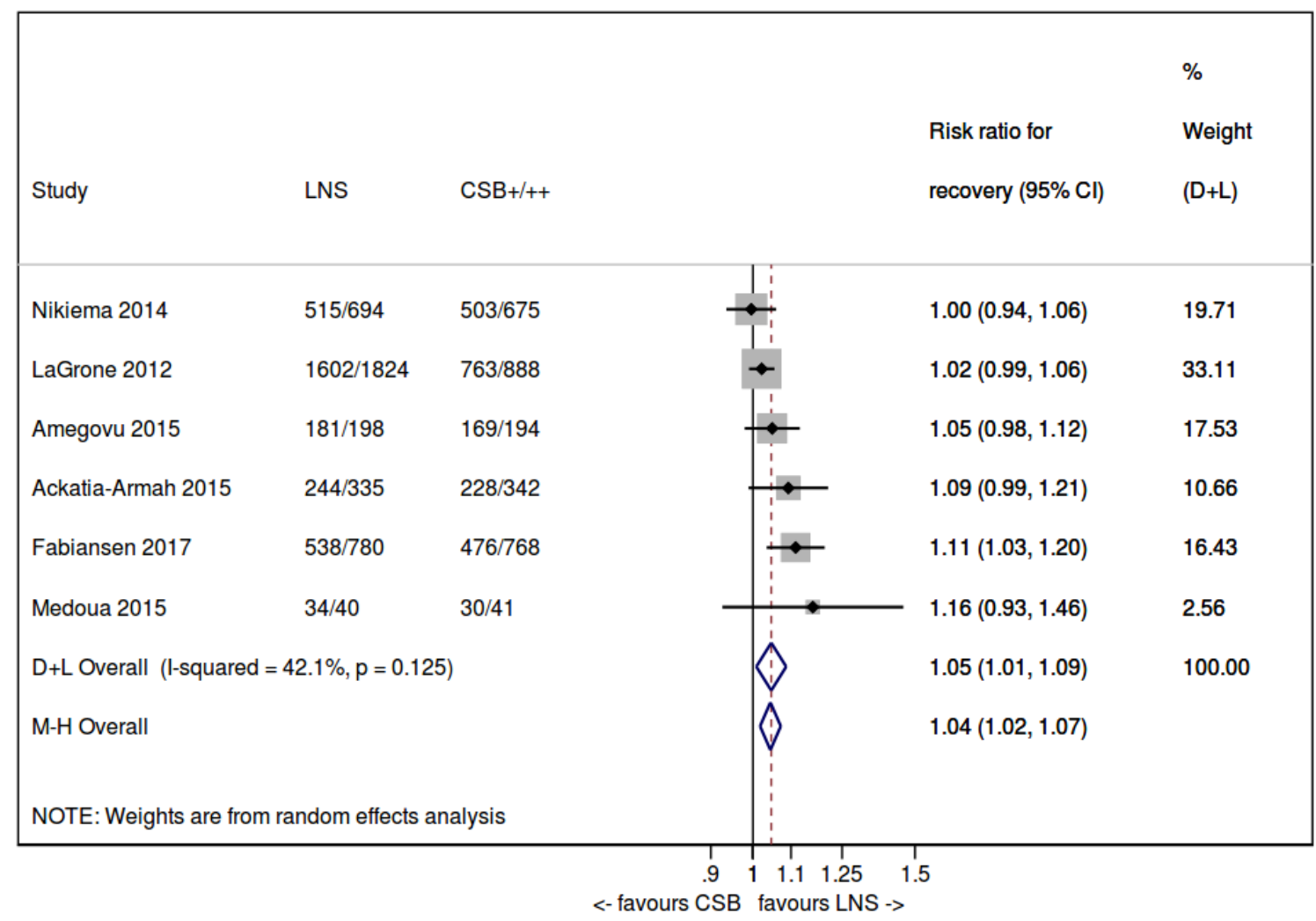

Abbreviations: CSB, corn-soy blend; LNS, lipid-based nutrient supplement 
medRxiv preprint doi: https://doi.org/10.1101/2021.01.16.21249861; this version posted January 20, 2021. The copyright holder for this preprint (which was not certified by peer review) is the author/funder, who has granted medRxiv a license to display the preprint in perpetuity.

It is made available under a CC-BY 4.0 International license .

Figure 3: A random effects meta-analysis of studies comparing the relative risk of defaulting in children treated with milk-protein LNS vs soy-based LNS

\begin{tabular}{|c|c|c|c|c|}
\hline \multirow[b]{3}{*}{ Study } & & & \multirow{3}{*}{$\begin{array}{l}\text { Risk ratio for } \\
\text { default }(95 \% \mathrm{Cl})\end{array}$} & \multirow{2}{*}{$\begin{array}{l}\% \\
\text { Weight }\end{array}$} \\
\hline & Milk-protein & Soy-based & & \\
\hline & LNS & LNS & & $(\mathrm{D}+\mathrm{L})$ \\
\hline Stobaugh 2016 & $16 / 1144$ & $28 / 1086$ & $0.54(0.30,1.00)$ & 49.13 \\
\hline LaGrone 2012 & $8 / 918$ & $14 / 906$ & $0.56(0.24,1.34)$ & 24.39 \\
\hline Matilsky 2009 & $10 / 456$ & $12 / 438$ & $0.80(0.35,1.83)$ & 26.48 \\
\hline \multicolumn{3}{|c|}{$D+L$ Overall (I-squared $=0.0 \%, p=0.746$ ) } & $0.61(0.40,0.93)$ & 100.00 \\
\hline $\mathrm{M}-\mathrm{H}$ Overall & & & $0.61(0.40,0.93)$ & \\
\hline NOTE: Weights & $\mathrm{n}$ random effe & alysis & & \\
\hline
\end{tabular}

Abbreviations: LNS, lipid-based nutrient supplement; MP, milk-protein; SB, soy-based 
medRxiv preprint doi: https://doi.org/10.1101/2021.01.16.21249861; this version posted January 20, 2021. The copyright holder for this preprint (which was not certified by peer review) is the author/funder, who has granted medRxiv a license to display the preprint in perpetuity.

It is made available under a CC-BY 4.0 International license .

Figure 4: A random effects meta-analysis comparing the probability of recovery in children with MAM treated with a food supplement vs nutrition education alone

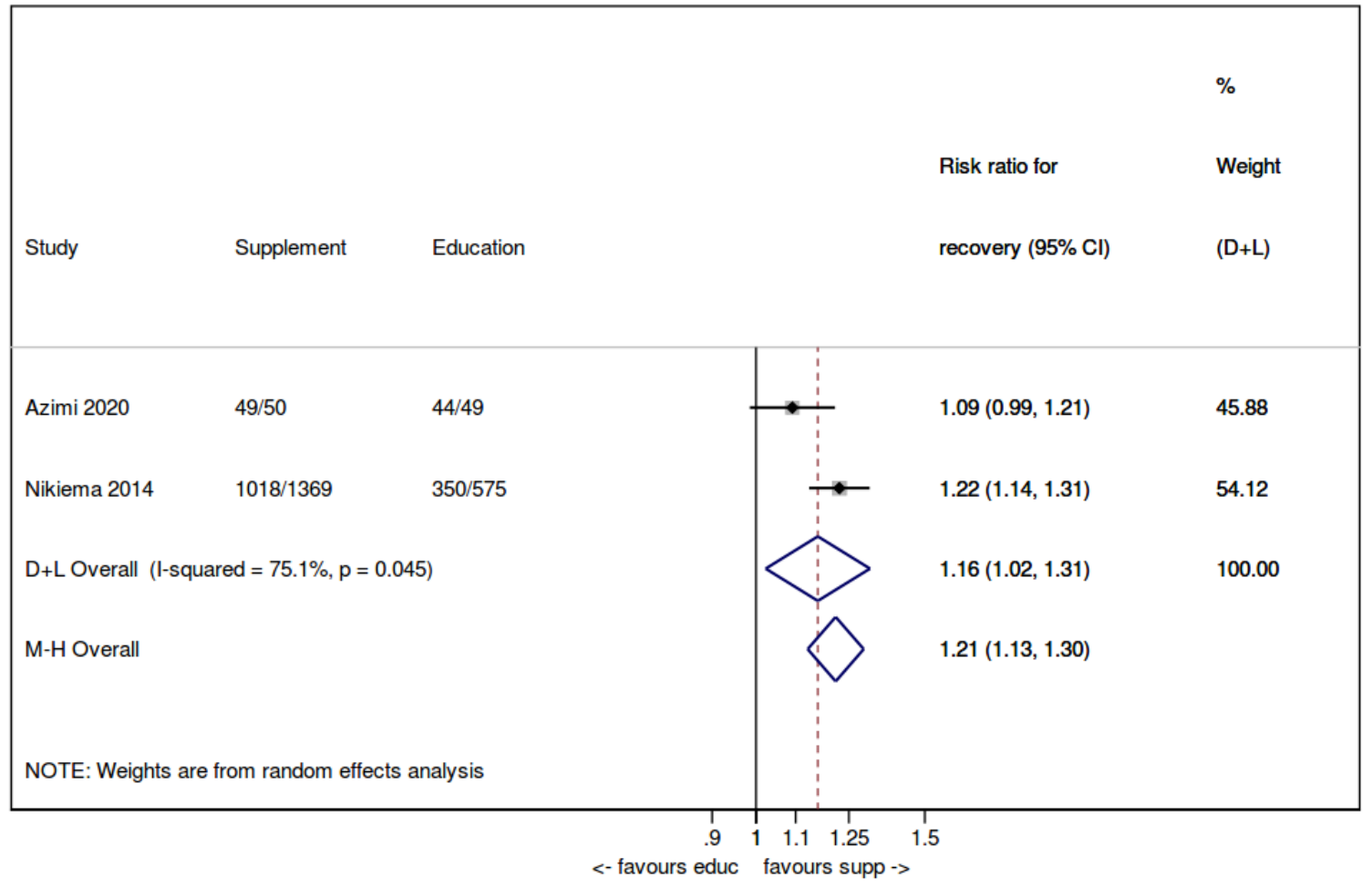

